\title{
UM ESTUDO DOS CONHECIMENTOS DE ALUNOS UNIVERSITÁRIOS EM RELAÇÃO AO SISTEMA ÚNICO DE SAÚDE.
}

A study of the knowledge of university students regarding the Health System.

http://dx.doi.org/10.21116/2016.2

\section{SPERANDIO, Ana Maria Girotti}

Faculdade Jaguariúna (FAJ) e Max Planck, Universidade Estadual de Campinas (UNICAMP)

\section{TORRES, Anelize Sgorlon Pinheiro}

Faculdade de Medicina de Jundiaí (FMJ) e Faculdade Jaguariúna (FAJ)

Resumo: O presente estudo trata-se de um artigo original, sendo uma pesquisa quantitativa com objetivo de demonstrar o conhecimento de base dos estudantes sobre funcionamento do Sistema Único de Saúde (SUS) anteriormente as aulas de Saúde Coletiva. Os dados foram coletados a partir da aplicação de um questionário dissertativo na disciplina de Saúde Coletiva para graduandos dos cursos de Enfermagem e Fisioterapia de uma Faculdade Privada no interior de São Paulo. Os resultados demonstram que os graduandos possuem entendimento que necessitam ser ampliados sobre 0 enfoque teórico e pratico, e acrescentam a necessidade de novos estudos com enfoque no ensino e pratica do campo da saúde publica.

Palavras chave: Sistema Único de Saúde, Saúde Pública, Enfermagem, Fisioterapia, Estudantes.

Abstract: The present study this is a unique, being a quantitative research in order to show the students the basic knowledge about functioning of the Unified Health System above the collective health classes. Data were collected from the application of a questionnaire dissertative in Public Health course for graduates of Nursing and Physiotherapy courses of a Private School in São Paulo. The results demonstrate that graduates have understanding that need to be expanded on theoretical approach and practice, and add the need for further research focusing on the teaching and practice of public health field.

Keywords: Health System, Public Health, Nursing, Physiotherapy, Students.

\section{INTRODUÇÃO}

No Brasil o Sistema Único de Saúde (SUS) culminou a partir Constituição Federal de 1988, que determinou ser dever do Estado garantir saúde a toda a população e, para tanto, criou o Sistema Único de Saúde. Em 
1990, o Congresso Nacional aprovou a Lei Orgânica da Saúde que detalha o funcionamento do Sistema, constituindo assim a maior política de inclusão social da história do Brasil (PORTAL SAÚDE, 2015).

A formação de estudantes na área da saúde atualmente está diretamente ligada às tendências do mercado de trabalho e induzidas em razão de questões sociais, culturais e políticas. Frente aos fatores citados os Ministérios da Saúde bem como da educação induzem que as formações sejam de acordo com as necessidades reais vivenciadas pela sociedade civil que utilizam o SUS (CECCIM \& FEUERWERKER, 2004).

Os profissionais da saúde presentes nos diferentes níveis de assistência são Enfermeiros, Fisioterapeutas, Nutricionistas, Dentistas, Fonoaudiólogos, Médicos e Psicólogos, estes trabalham diretamente com o paciente, sendo fundamental que o processo de formação acadêmico seja de maneira teórico-prático dentro dos serviços de saúde públicos (AL et al, 2009).

A partir da Lei 8080 no artigo no 27 referente às novas Diretrizes Curriculares Nacionais, trouxe a participação da prática de ensino e pesquisa nos serviços públicos, decorrentes de visitas, estágios supervisionados, bem como projetos de extensões (SERIANO et al,2013). Isto se tornou um avanço diferencial para que os graduandos pudessem aplicar conhecimentos teóricos junto a prática assistencial oferecida aos usuários destes serviços.

No que se diz a respeito às Diretrizes Curriculares Nacionais (2011) referentes ao curso de Enfermagem destaca-se "Parágrafo Único. A formação do Enfermeiro deve atender as necessidades sociais da saúde, com ênfase no Sistema Único de Saúde (SUS) e assegurar a integralidade da atenção e a qualidade e humanização do atendimento".

O SUS não dever ser simplesmente um campo de estágio para aprendizagem prática, mas sim um interlocutor das instituições de ensino a partir de questões relacionadas a gestão dos serviços, assim estabelecendo elaboração e implantando politicas vinculadas a formação profissional (NUNES, 2006).

No entanto muitas escolas e faculdades privadas com curso de enfermagem estão sendo criadas desordenadamente, estabelecendo somente 
critérios mínimos para funcionamento, isto acarreta na formação de profissionais desqualificados para o mercado de trabalho.

De acordo com SENA \& SILVA (2011) a Enfermagem no Brasil está diretamente ligada as questões políticas devido ao crescente número de destes profissionais demonstrando uma solidificação da classe nos setores do SUS, colaborando sistematicamente com o sistema, visto que tem estabelecido questões referentes a formação dos graduandos e produções culminadas a partir do conhecimento deste.

Em consonância aos autores citados acima, COSTA \& MIRANDA (2009) relatam as alterações que vem sendo ocorrido quanto ao ensino de enfermagem, qual o currículo mínimo é aprovado pelo conselho Federal de Educação sem a liberação da Associação Brasileira de Enfermagem (ABEn). De um modo geral a classe profissional predominantemente nos setores de saúde é o da Enfermagem composta por auxiliares, técnicos e Enfermeiros, a atuação deste é determinante nas questões referentes aos cuidados gerais do paciente, iniciando pelo acolhimento, prevenção, promoção e reabilitação.

A atuação dos profissionais fisioterapeutas esta concomitantemente aos dos profissionais enfermeiros nos serviços de saúde, sendo uma comunicação ativa e atuante nos cuidados com o paciente.

No Brasil outros cursos relacionados a área da saúde como o curso de Fisioterapia também está ligado à prática curativa, mas o Conceito de Saúde Coletiva é amplo, diversificado, no qual a educação permanente é presente num contexto geral sobre saúde. Condizendo com as Diretrizes Curriculares que pontam "a formação do fisioterapeuta deve contentar as necessidades sociais da saúde, com ênfase no SUS" (CONSELHO NACIONAL DE EDUCAÇÃO, 2001).

O domínio do conhecimento técnico é indispensável, porém não é suficiente para a construção da relação teórica e pratica no campo da saúde (BACKES et al, 2014), para além da formação específica, espera-se que o profissional com formação de saúde compreenda de forma crítica e reflexiva as políticas que norteiam o campo da saúde. 


\section{Objetivo da Pesquisa}

O objetivo deste artigo é demonstrar e descrever o conhecimento em relação ao Sistema único de saúde dos alunos ingressantes nos cursos de Enfermagem e Fisioterapia da Faculdade de Jaguariúna entre 2012 e 2014.

\section{METODOLOGIA}

Este artigo trata-se de um artigo original descritivo e quantitativo, 0 estudo ocorreu na Faculdade de Jaguariúna, uma faculdade privada, que em 2015 que tem crescido nos últimos anos, em relação ao numero de alunos e sua estrutura física.

Para atender a pesquisa deste estudo foi elaborado um questionário (anexo1) em uma disciplina ministrada Universidade Estadual de Campinas (UNICAMP) em 2008 e readaptado para a disciplina de Saúde Coletiva I da Faculdade Jaguariúna.

O questionário composto por seis questões dissertativas de conhecimento básico em relação ao SUS, esse aplicado durante uma atividade em sala de aula contemplada no plano de estudo da disciplina de Saúde Coletiva I para alunos de Enfermagem e Fisioterapia no primeiro dia de aula, período 2012 a 2014. Esta disciplina e ministrada anualmente no segundo semestre, esta disciplina tem uma carga horária de 64 horas.

Para analise quantitativa do estudo foi utilizado o programa EPI INFO 7 respeitando-se todas orientações do programa. Para este artigo foram considerados quatrocentos (400) questionários preenchidos do total de cerca de seiscentos (600), e os demais ainda estão em estudos.

Os dados compilados foram apresentados em forma de gráficos, de modo quantitativo considerando percentuais de acordo com as respostas obtidas das questões.

Não houve critérios de exclusão dos questionários.

\section{RESULTADOS E DISCUSSÕES}


A disciplina de Saúde Coletiva faz parte da nova grade curricular da saúde tornando-se fundamental, por exemplo, nos cursos Fisioterapia e Enfermagem na Faculdade Jaguariúna foi constatada por meio da pesquisa que era o primeiro contato de entendimento do funcionamento do Sistema Único de saúde (SUS), por questões sociais, culturais e educacionais.

A saúde coletiva relacionada ao ser humano não se trata somente das questões orgânicas e biológicas, mas de sua colocação frente a situações de caráter social, ético, político em um contexto histórico, este fundamental durante a construção do profissional da área da saúde (BISCARDE et al, 2014).

Os resultados foram apresentados pelos gráficos produzidos através de uma máscara elaborada no Programa EPI INFO7.

\section{Análise das questões investigadas.}

A fim de se obter resultados gerais, os resultados por questão não foram subdivididos em curso, considerando totais como área da saúde. A aplicação dos questionários representado no quadro 1 totalizaram quatrocentos (400) questionários respondidos, sendo trezentos e dezoito (318) para alunos de Fisioterapia e oitenta e dois (82) para os alunos Enfermagem.

Quadro 1. Número total de questionários aplicados.

\begin{tabular}{|l|r|r|r|r|r|r|}
\hline \multicolumn{1}{|c|}{ Curso } & Frequency & Percent & Cum. Percent & 95\% CI Lower & 95\% CI Upper & \\
\hline Enfermagem & 82 & $20,50 \%$ & $20,50 \%$ & $16,72 \%$ & $24,86 \%$ & \\
\hline Fisioterapia & 318 & $79,50 \%$ & $100,00 \%$ & $75,14 \%$ & $83,28 \%$ & \\
\hline TOTAL & $\mathbf{4 0 0}$ & $\mathbf{1 0 0 , 0 0} \%$ & $\mathbf{1 0 0 , 0 0 \%}$ & & & \\
\hline
\end{tabular}

A primeira questão aplicada como demostrada no Quadro 2 infere que $90 \%$ sabem a respeito da sigla SUS. No entanto o segundo resultado aponta que aproximadamente $8 \%$ dos alunos "Não Sabe". 
Quadro 2. Questão 1- O que é o SUS?

\begin{tabular}{|c|c|c|c|c|c|}
\hline 1-O que é o SUS? & Frequency & Percent & Cum. Percent & 95\% CI Lower & 95\% CI Upper \\
\hline Não Sabe & 20 & $5,00 \%$ & $5,00 \%$ & $3,16 \%$ & $7,74 \%$ \\
\hline Orgão de saúde pública & 14 & $3,50 \%$ & $8,50 \%$ & $2,00 \%$ & $5,94 \%$ \\
\hline Sistema de saúde pública & 15 & $3,75 \%$ & $12,25 \%$ & $2,19 \%$ & $6,25 \%$ \\
\hline Sistema Único de Saúde & 345 & $86,25 \%$ & $98,50 \%$ & $82,39 \%$ & $89,39 \%$ \\
\hline Sistema Unificado de Saúde & 2 & $0,50 \%$ & $99,00 \%$ & $0,09 \%$ & $2,00 \%$ \\
\hline Um tipo de Convenio & 4 & $1,00 \%$ & $100,00 \%$ & $0,32 \%$ & $2,72 \%$ \\
\hline TOTAL & 400 & $100,00 \%$ & $100,00 \%$ & & \\
\hline
\end{tabular}

Conforme apresentado verifica-se um desafio para as Instituições de Ensino Superior para propiciar informações, conhecimento e responsabilidade na educação destes futuros profissionais (GONZÁLEZ \& ALMEIDA, 2010). Para construção de um modelo que não esteja somente compreendido no conhecimento técnico de funcionamento, mas também observando, a fim de corroborar com a elaboração do pensamento crítico a respeito de políticas públicas e saúde vinculando ao desenvolvimento profissional (Pinheiro et al, 2009)

Quadro 3.Questão 2- O que vem a sua cabeça quando o senhor ouve a palavra SUS? 


\begin{tabular}{|c|c|c|c|c|c|}
\hline 2-O que vem a sua cabeça quando o senhor ouve a palavra SUS? & Frequency & Percent & Cum. Percent & 95\% Cl Lower & 95\% Cl Upper \\
\hline Centro de atendimento medico & 16 & $4,00 \%$ & $4,00 \%$ & $2,38 \%$ & $6,55 \%$ \\
\hline Corrupção & 9 & $2,25 \%$ & $6,25 \%$ & $1,10 \%$ & $4,38 \%$ \\
\hline Demora & 57 & $14,25 \%$ & $20,50 \%$ & $11,05 \%$ & $18,15 \%$ \\
\hline Desrespeito & 83 & $20,75 \%$ & $41,25 \%$ & $16,95 \%$ & $25,13 \%$ \\
\hline Direito de todos em território brasileiro independentemente da classe social e idade & 2 & $0,50 \%$ & $41,75 \%$ & $0,09 \%$ & $2,00 \%$ \\
\hline Hospital & 19 & $4,75 \%$ & $46,50 \%$ & $2,96 \%$ & $7,45 \%$ \\
\hline Hospital publico & 22 & $5,50 \%$ & $52,00 \%$ & $3,56 \%$ & $8,33 \%$ \\
\hline Humildes, pobres & 7 & $1,75 \%$ & $53,75 \%$ & $0,77 \%$ & $3,73 \%$ \\
\hline Não Sabe & 10 & $2,50 \%$ & $56,25 \%$ & $1,28 \%$ & $4,70 \%$ \\
\hline Plano de saúde & 3 & $0,75 \%$ & $57,00 \%$ & $0,19 \%$ & $2,36 \%$ \\
\hline Programa do governo & 9 & $2,25 \%$ & $59,25 \%$ & $1,10 \%$ & $4,38 \%$ \\
\hline Saúde & 23 & $5,75 \%$ & $65,00 \%$ & $3,76 \%$ & $8,63 \%$ \\
\hline Sem resposta & 2 & $0,50 \%$ & $65,50 \%$ & $0,09 \%$ & $2,00 \%$ \\
\hline Sistema falho & 117 & $29,25 \%$ & $94,75 \%$ & $24,89 \%$ & $34,02 \%$ \\
\hline Sistema Público de Saúde & 21 & $5,25 \%$ & $100,00 \%$ & $3,36 \%$ & $8,04 \%$ \\
\hline TOTAL & 400 & $100,00 \%$ & $100,00 \%$ & & \\
\hline
\end{tabular}

Estes resultados obtidos na questão 2, observadas no quadro 3, permite um questionamento quanto a infraestrutura, recursos e qualificações dos profissionais atuantes no SUS.

Estudos realizados indicam profissionais que trabalham no SUS desconhecem as diretrizes e princípios que os regem, também relatam a demora e a necessidade de um novo modo de gerir, antagonicamente estes falam do SUS como um modelo para outros países, transmitindo culturalmente o habito da reclamação (BACKES et al, 2014).

Estes fatores trazem à tona a importância destes profissionais se qualificarem de forma teórico-prático, assim como os futuros enfermeiros devem ter formações que os instruem coerentemente com o modelo assistencial, junto às habilidades de gerenciamento, a partir de instituições de ensino que estejam dispostas a mudanças a fim de trazer melhorias à qualidade do profissional concomitantemente a saúde prestada. A partir de diferentes metodologias, bem como projeto políticos e pedagógicos (COSTA \& MIRANDA, 2009).

Isto é demonstrado por Biscarde et al (2014) que ressaltam a relação entre os profissionais e usuários, cada qual contribuindo com suas experiências e conhecimento, otimizando a assistência no período entre espera e 
atendimento ao invés de uma única das partes contribuírem para funcionamento do SUS.

Quadro 4. Questão 3- Quem tem direito ao SUS?

\begin{tabular}{|c|c|c|c|c|c|c|}
\hline 3-Quem tem direito ao SUS? & Frequency & Percent & Cum. Percent & 95\% Cl Lower & 95\% Cl Upper & \\
\hline Estrangeiros em território brasileiro & 1 & $0,25 \%$ & $0,25 \%$ & $0,01 \%$ & $1,61 \%$ & \\
\hline Não pussui convênio & 3 & $0,75 \%$ & $1,00 \%$ & $0,19 \%$ & $2,36 \%$ & \\
\hline Não Sabe & 4 & $1,00 \%$ & $2,00 \%$ & $0,32 \%$ & $2,72 \%$ & \\
\hline Pessoas sem condições financeiras principalmente crianças e idosos & 20 & $5,00 \%$ & $7,00 \%$ & $3,16 \%$ & $7,74 \%$ & 】 \\
\hline Pessoas sem condições principalmente crianças e idosos & 2 & $0,50 \%$ & $7,50 \%$ & $0,09 \%$ & $2,00 \%$ & \\
\hline Sem Resposta & 1 & $0,25 \%$ & $7,75 \%$ & $0,01 \%$ & $1,61 \%$ & \\
\hline Toda população & 41 & $10,25 \%$ & $18,00 \%$ & $7,54 \%$ & $13,75 \%$ & \\
\hline Todo brasileiro ou estrangeiro que esteja no Brasil & 8 & $2,00 \%$ & $20,00 \%$ & $0,93 \%$ & $4,06 \%$ & I \\
\hline Todo cidadão cadastrado no SUS & 16 & $4,00 \%$ & $24,00 \%$ & $2,38 \%$ & $6,55 \%$ & | \\
\hline Todos & 158 & $39,50 \%$ & $63,50 \%$ & $34,71 \%$ & $44,50 \%$ & \\
\hline Todos cidadãos brasileiros & 46 & $11,50 \%$ & $75,00 \%$ & $8,62 \%$ & $15,14 \%$ & \\
\hline Todos cidadãos com endereço fixo e documento & 1 & $0,25 \%$ & $75,25 \%$ & $0,01 \%$ & $1,61 \%$ & \\
\hline Todos nascidos no Brasil & 5 & $1,25 \%$ & $76,50 \%$ & $0,46 \%$ & $3,06 \%$ & \\
\hline Todos os cidadãos & 81 & $20,25 \%$ & $96,75 \%$ & $16,49 \%$ & $24,60 \%$ & \\
\hline Todos que necessitam de saúde publica & 10 & $2,50 \%$ & $99,25 \%$ & $1,28 \%$ & $4,70 \%$ & | \\
\hline Todos que pagam impostos & 3 & $0,75 \%$ & $100,00 \%$ & $0,19 \%$ & $2,36 \%$ & \\
\hline TOTAL & 400 & $100,00 \%$ & $100,00 \%$ & & & \\
\hline
\end{tabular}

No contexto geral $85 \%$ foram assertivos com a resposta, ainda sim observa-se o desconhecimento da Lei 8080 - Lei orgânica da saúde e 
recentemente sobre a Portaria MS/GM nำ 940, de 28/04/2011 responsável pela regulamentação do Sistema Cartão Nacional de Saúde, o qual se trata de uma sabe de dados com a finalidade de identificar os usuários do SUS, saúde suplementar e domicílio. O Cartão Nacional de Saúde também é emitido para brasileiros que residem no exterior e estrangeiros que não residem no Brasil, mas estejam no território (RIO PRETO, 2015).

Quadro 5. Questão 4- Existe diferença entre centro de saúde ou Hospital? Se sim, qual?

\begin{tabular}{|c|c|c|c|c|c|c|}
\hline 4-Existe diferença entre centro de saúde ou hospital? Se sim, qual? & Frequency & Percent & Cum. Percent & 95\% Cl Lower & 95\% Cl Upper & \\
\hline Não & 24 & $6.00 \%$ & $6,00 \%$ & $3,96 \%$ & $8,92 \%$ & 】 \\
\hline Não Sabe & 63 & $15,75 \%$ & $21,75 \%$ & $12,40 \%$ & $19,78 \%$ & $\square$ \\
\hline Sem Resposta & 1 & $0,25 \%$ & $22,00 \%$ & $0,01 \%$ & $1,61 \%$ & \\
\hline Sim, centro de saúde atende casos especificos e hospital atende emergência & 56 & $14,00 \%$ & $36,00 \%$ & $10,83 \%$ & $17,88 \%$ & $\square$ \\
\hline Sim, centro de saúde atendimento primário, prevenção e praticas de saúde e hospital para urgência e emergência & 110 & $27,50 \%$ & $63,50 \%$ & $23,24 \%$ & $32,20 \%$ & \\
\hline Sim, centro de saúde para pequenos casos e hospital para casos mais especificos & 38 & $9,50 \%$ & $73,00 \%$ & $6,89 \%$ & $12,91 \%$ & - \\
\hline Sim, centro de saúde previne a doença e hospital trata a doença & 18 & $4,50 \%$ & $77,50 \%$ & $2,77 \%$ & $7,15 \%$ & I \\
\hline Sim, centro de saúde procedimentos mais simples e hospital procedimentos avançados & 90 & $22,50 \%$ & $100,00 \%$ & $18,56 \%$ & $26,97 \%$ & \\
\hline TOTAL & 400 & $100,00 \%$ & $100,00 \%$ & & & \\
\hline
\end{tabular}

O SUS possui princípios, os quais podem cita-los: integralidade, universalidade e equidade, esses estão presentes em todos os níveis de assistência. $O$ quadro 5 demonstra uma linha tênue entre o entendimento entra Centro de Saúde e Hospitais, que seria o atendimento de urgência e emergência encontrados nos Hospitais (Souza et al, 2013), abrangendo em torno de $33 \%$, em sequencia $27 \%$ diferenciam entre "procedimentos simples e procedimentos avançados" e $20 \%$ "Não Sabe", mesmo em algum momento da vida ter utilizado algum destes.

É de suma importância ressaltar que nesses níveis de assistência encontram-se diferentes classes e profissionais da área da saúde que atuam de maneira multidisciplinar em suas especialidades e especificidades. 
Estudos relatam que a partir de experiências feitas por estudantes de medicina no SUS, um grupo seleto evidenciou questões referentes a infraestrutura, o atendimento entre médico e paciente, a aplicações teóricas postas em prática (SOUZA et al, 2013).

Em outros estudos relatam que cirurgiões dentistas ao realizarem seus estágios extracurriculares no SUS possibilitaram a estes profissionais interesses do funcionamento em atenção primaria, transmitindo autonomia e resolutividade de questões junto a outros profissionais da saúde que atuam nestes estabelecimentos (CIUFFO \& RIBEIRO, 2008).

No que se diz aos alunos de Farmácia questiona-se sobre a assistência farmacêutica no SUS, porém está ainda não se tem uma consideração, nem reconhecimentos necessários para que os profissionais possam atuar, com dificuldade de compreensão de sua função de forma integral no SUS, isto devido aos gestores e as instituições não oferecerem subsídios para o desenvolvimento do trabalho, bem como as não abordarem corretamente o conhecimento necessário para cientes destes (NICOLINE \& VIERA, 2011).

A Nutrição também esta em evidencia dentre as profissionais de saúde operantes no SUS, integrando os usuários o aporte nutricional necessário, concomitantemente aos outros profissionais (MEDEIROS et al, 2014).

Quanto aos alunos de fisioterapia, um estudo estabelece através de percentagem de que parte que cursam entre o $6^{\circ}$ e $8^{\circ}$ período se dizem não condizentes para atuação no SUS, diferentemente dos quais estão no último ano que estabelecem um maior número de horas de aula quanto de estágio em campo, disseram-se adequados para exercer a profissão no SUS. Mas quanto as teorias aplicação SUS, alunos de uma Instituição de Ensino de Fortaleza mostraram-se condizentes a partir do $4^{\stackrel{0}{ }}$ período devido suas grades curriculares contemplarem disciplinas que possuem a ementa sobre doutrinas, relações organizações e a participação da sociedade civil (SERIANO et al, 2013). 
Quadro 6. O que você tinha quando foi ao hospital a última vez?

\begin{tabular}{|c|c|c|c|c|c|}
\hline 5- 0 que você tinha quando foi ao hospital a ultima vez? & Frequency & Percent & Cum. Percent & 95\% C Lower & 95\% C Upper \\
\hline Algias & 46 & $11,50 \%$ & $11,50 \%$ & $8,62 \%$ & $15,14 \%$ \\
\hline Caso clínico & 67 & $16,75 \%$ & $28,25 \%$ & $13,30 \%$ & $20,86 \%$ \\
\hline Cirurgia & 15 & $3,75 \%$ & $32,00 \%$ & $2,19 \%$ & $6,25 \%$ | \\
\hline Conjuntivite & 3 & $0,75 \%$ & $32,75 \%$ & $0,19 \%$ & $2,36 \%$ \\
\hline Dores abdominais & 14 & $3,50 \%$ & $36,25 \%$ & $2,00 \%$ & $5,94 \%$ | \\
\hline Exames de imagem & 6 & $1,50 \%$ & $37,75 \%$ & $0,61 \%$ & $3,40 \%$ \\
\hline Febre & 10 & $2,50 \%$ & $40,25 \%$ & $1,28 \%$ & $4,70 \%$ | \\
\hline Fraturas & 15 & $3,75 \%$ & $44,00 \%$ & $2,19 \%$ & $6,25 \%$ | \\
\hline Hipertensão & 11 & $2,75 \%$ & $46,75 \%$ & $1,45 \%$ & $5,01 \%$ | \\
\hline Infecção & 26 & $6,50 \%$ & $53,25 \%$ & $4,37 \%$ & $9,50 \%$ \\
\hline Inflamação & 24 & $6,00 \%$ & $59,25 \%$ & $3,96 \%$ & $8,92 \%$ | \\
\hline Intoxicação & 2 & $0,50 \%$ & $59,75 \%$ & $0,09 \%$ & $2,00 \%$ \\
\hline Luxação & 1 & $0,25 \%$ & $60,00 \%$ & $0,01 \%$ & $1,61 \%$ \\
\hline Não Sabe & 21 & $5,25 \%$ & $65,25 \%$ & $3,36 \%$ & $8,04 \%$ \\
\hline Outros & 30 & $7,50 \%$ & $72,75 \%$ & $5,20 \%$ & $10,65 \%$ \\
\hline Parto & 21 & $5,25 \%$ & $78,00 \%$ & $3,36 \%$ & $8,04 \%$ \\
\hline Reação anafilática & 11 & $2,75 \%$ & $80,75 \%$ & $1,45 \%$ & $5,01 \%$ | \\
\hline Sintomas de gripe & 35 & $8,75 \%$ & $89,50 \%$ & $6,25 \%$ & $12,07 \%$ \\
\hline Traumas acidentais & 15 & $3,75 \%$ & $93,25 \%$ & $2,19 \%$ & $6,25 \%$ | \\
\hline Virose & 27 & $6,75 \%$ & $100,00 \%$ & $4,58 \%$ & $9,79 \%$ \\
\hline TOTAL & 400 & $100,00 \%$ & $100,00 \%$ & & \\
\hline
\end{tabular}


Nesta questão foram estabelecidas as respostas frequentes e forma abrangente, observou-se a predominância dos "casos clínicos" em torno de $21 \%$, casos que são necessárias investigações clínicas, coleta de exames laboratoriais e exames de imagem, seguido por "queixas álgicas" em 16\%, sem especificidades.

Estudos demonstram que casos de doença são as causas de procuras recorrentes dos usuários ao SUS (RIBEIRO et al, 2003), ligados a população baixa renda, provocando a necessidade de articulação deste para realização da promoção e prevenção da saúde.

Por fim na Questão 6 "O que você faz para se manter saudável?", os alunos que participaram desta pesquisa indicam a alimentação concomitante aos exercícios físicos como a principal ação para manter-se saudável aderindo próximo a $44 \%$

Porém o resultado "nada" também se mostrou significativo abrangendo $17 \%$, isso pode enfatizar a falha da promoção, prevenção da saúde.

Após a relação de respostas evidenciadas nas questões apresentadas acima, verifica-se a relevância das formações de profissionais da saúde endossada pela disciplina de Saúde Coletiva I, considerando uma perspectiva voltada para o entendimento do SUS.

\section{CONSIDERAÇÕES FINAIS}

O tempo de existência do SUS exacerba a necessidade dos estudantes de Ensino Superior realizarem pesquisas e estágios em diferentes atendimentos oferecidos por este. Desta forma os estudantes devem desenvolver competências gerenciais adequadas proporcionadas pela graduação, estabelecendo o enlace entre teoria e a prática.

A relação entre a Instituição e o SUS possibilita a multidisciplinaridade, bem como a intersetorialidade, trazendo os graduandos da área da saúde para atuarem de forma cabível, correspondendo com o serviço prestado aos usuários deste sistema.

Para continuidade de uso deste questionário como instrumento de pesquisa sugere-se elaboração de novas questões para serem acrescentadas 
e também reformulações dessas questões para serem de característica fechada, a partir dos resultados obtidos neste artigo.

\section{REFERÊNCIAS BIBLIOGRÁFICAS}

BACKES, D.S. et al. O Sistema Único de Saúde idealizado versus o realizado: contribuições da Enfermagem. Rev. Latino-Am. Enfermagem. v.22, n.6, p.1026-1033, 2014.

BISCARDE, D.G.S.; PEREIRA-SANTOS M.; SILVA, L.B. Formação em saúde, extensão universitária e Sistema Único de Saúde (SUS): conexões necessárias entre conhecimento e intervenção centradas na realidade e repercussões no processo formativo. Interface. v.18, n.48, p.177-186, 2014.

CECCIM, R.B; Feuerwerker, L.C.M.. Quadrilátero da Formação para a Área da Saúde: Ensino, Gestão, Atenção e Controle Social PHYSIS: Rev. Saúde Coletiva, Rio de Janeiro, v.14, n.1, p.41- 65, 2004.

CIUFFO, R.S.; RIBEIRO, V,M. Sistema Único de Saúde e a formação dos médicos: um diálogo possível?. Interface. v.12, n.24, p.125-140,2008.

Conselho Nacional de Educação. Diretrizes curriculares nacionais dos cursos de graduação em Enfermagem, Medicina e Nutrição. Brasília; 2001.

Conselho Nacional de Educação. Diretrizes curriculares nacionais dos cursos de graduação em fisioterapia, fonoaudiologia e terapia ocupacional. Brasília; 2001.

COSTA, R.K.S.; MIRANDA, F.A.N. Sistema Único de Saúde e da família na formação acadêmica do enfermeiro. Rev. bras. enferm. v.62, n.2, p.300-304, 2009.

ERDMANN, A.L. et al. O olhar dos estudantes sobre sua formação profissional para o Sistema Único de Saúde, Acta Paul Enferm. v.22, n.3, p.288-94, 2009.

GONZÁLEZ, A.D.; ALMEIDA, M.J. Integralidade da saúde - norteando mudanças na graduação dos novos profissionais. Ciência \& Saúde Coletiva. v.15, n.3,p.757-762, 2010.

MEDEIROS, M.A.T. et al. A integralidade como eixo da formação em proposta interdisciplinar: estágios de Nutrição e Psicologia no campo da Saúde Coletiva. Rev. Nutr. v.27, n.6, p.785-798,2014.

NICOLINE, C.B.I.; VIEIRA, R.C.P.A. Assistência farmacêutica no Sistema Único de Saúde (SUS): percepções de graduandos em Farmácia. Interface. v.15, n.39, p.1127-1144,2011. 
NUNES, E.D. Saúde coletiva: uma história recente de um passado remoto. In: Campos GWS, et al (editores). Tratado de saúde coletiva. 2a ed. São Paulo: Hucitec; p.19-40,2006.

PINHEIRO et al. Conhecimento de graduandos em Fisioterapia na Universidade de Fortaleza sobre o Sistema Único de Saúde. Fisioterapia e Pesquisa, São Paulo. v.16, n.3, p.211-6, 2009.

Portal Saúde [Internet]. Histórico: Do Sanitarismo a Municipalização.

Disponível em: http://portalsaude.saude.gov.br/index.php/o-ministerio/historico. Acesso em: 27 jun. 2015.

RIBEIRO, M.C.S.A. et al. Perfil sócio demográfico e padrão de utilização de serviços de saúde para usuários e não-usuários do SUS.PNAD. 2003.

RIO PRETO. Cartão Sus (Cartão Nacional de Saúde). Disponível em: http://gestao.saude.riopreto.sp.gov.br/transparencia/modules/mastop publish/?t ac=Serv Cart SUS. Acesso em: 27 jun 2015.

SENA, R.R.; SILVA, K.L. A enfermagem como parceira solidária do Sistema Único de Saúde. Rev. esc. enferm. USP. v. 45, n.2, p.1792-1796, 2011. SERIANO, K.N.; MUNIZ, V.R.C.; DECARVALHO, M.I.M. Percepção de estudantes do curso de fisioterapia sobre sua formação profissional para atuação na atenção básica no Sistema Único de Saúde. Fisioter. Pesqui. v.20, n.3, p.250-255, 2013.

SOUZA, C.F.T. et al. A atenção primária na formação médica: a experiência de uma turma de medicina. Rev. bras. educ. med. v.37, n.3, p. 448-454,2013. 


\section{Anexo 1}

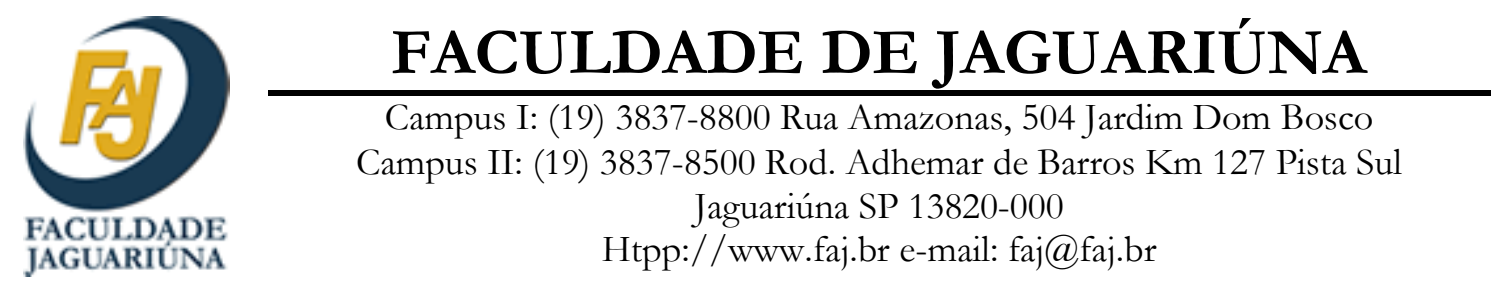

\section{DISCIPLINA SAÚDE COLETIVA I}

Profa. Dra. Ana Maria Girotti Sperandio

Professora Titular

Por favor, respondam essas questões ${ }^{1}$ abaixo:

\footnotetext{
${ }^{1}$ Questionário utilizado pelos alunos da FCM/UNICAMP em 2005 para desenvolvimento de pesquisa “Oi Calouro você conhece o SUS?".
} 
Questão 1: O que é SUS?

Questão 2: O que vem a sua cabeça quando o senhor ouve a palavra SUS?

Questão 3: Quem tem direito ao SUS?

Questão 4: Existe diferença entre Centro de Saúde e hospital? Qual?

Questão 5: O que o você tinha quando foi ao hospital pela última vez?

Questão 6: O que o você faz para se manter saudável? 


\section{SPERANDIO, Ana Maria Girotti}

Assessora Acadêmica da FAJ, Coordenadora do Núcleo de Estudos e Pesquisas Interdisciplinares (NEPI), Coordenadora do Grupo de Estudos de Mobilidade e Acessibilidade para Cidades Saudáveis, Pesquisadora do Laboratório de Investigações Urbanas (LABINUR), Professora Convidada da Faculdade de Engenharia, Arquitetura e Urbanismo/UNICAMP, Pesquisadora Líder do Grupo de Pesquisa de Planejamento Urbano Saudável (GPLUS) FEC/UNICAMP. Contato: amgspera@uol.com.br

\section{TORRES, Anelize Sgorlon Pinheiro}

Enfermeira, Especialista em Gestão estratégicas em Saúde e Segurança do Trabalho, Voluntária do Projeto MASSUr (Mobilidade e Acessibilidade Sustentáveis em saúde Urbana) FAJ/University of Michigan e Mestranda pela Faculdade de Medicina de Jundiaí. Contato: anesgorlon@hotmail.com 
